\title{
Vertical Scanning Interferometry: A New Method to Quantify Re- /De-Mineralization Dynamics of Dental Enamel
}

\author{
Isabella Pignatelli ${ }^{a}$, Aditya Kumar ${ }^{a}$, Kumar Shah $^{b}$, Magdalena Balonis $^{c, d, e}$, Mathieu Bauchy ${ }^{f}$, \\ Benjamin $W u^{b, c, e, g, *}$, Gaurav Sant ${ }^{a, g, *}$ \\ ${ }^{a}$ Laboratory for the Chemistry of Construction Materials $\left(L C^{2}\right)$, Department of Civil and Environmental Engineering, University \\ of California, Los Angeles, CA 90095, USA \\ ${ }^{b}$ Division of Advanced Prosthodontics, School of Dentistry, University of California Los Angeles, CA 90095 \\ ${ }^{c}$ Department of Materials Science and Engineering, University of California, Los Angeles, CA, USA \\ ${ }^{d}$ Institute for Technology Advancement (ITA), University of California, Los Angeles, CA, USA \\ ${ }^{e}$ Department of Bioengineering, University of California, Los Angeles, CA 90095 \\ ${ }^{f}$ Physics of Amorphous and Inorganic Solids Laboratory (PARISlab), Department of Civil and Environmental Engineering, \\ University of California, Los Angeles, CA 90095 \\ ${ }^{g}$ California Nanosystems Institute (CNSI), University of California, Los Angeles, CA 90095
}

\begin{abstract}
Remineralization and demineralization are processes that compete in the oral environment. At this time, numerous therapeutic agents are being developed to promote remineralization (precipitation) or suppress demineralization (dissolution). To evaluate the relative efficacy of such treatments, there is a need for non-invasive, real-time, high-resolution quantifications of topographical changes occurring during demineralization and remineralization. Vertical scanning interferometry (VSI) is demonstrated to be a quantitative method to assess reactions, and topographical changes occurring on enamel surfaces following exposure to demineralizing, and remineralizing liquids. First, the dissolution rate of enamel was compared to that of synthetic hydroxyapatite (HAP) under acidic conditions $(\mathrm{pH}=4)$. Second, VSI was used to compare the remineralization effects of $\mathrm{F}^{-}$-based and $\mathrm{CCP}$-ACP agents. The former produced a remineralization rate of $\approx 349 \mathrm{~nm} / \mathrm{h}$, similar to simulated body fluid (SBF; concentration $4.6 \mathrm{x}$ ) while the latter produced a remineralization rate of $\approx 55 \mathrm{~nm} / \mathrm{h}$, corresponding to $1.7 \mathrm{x} \mathrm{SBF}$. However, the precipitates formed by the CCP-ACP agent are found to demineralize $2.7 \mathrm{x}$ slower than that produced by its $\mathrm{F}^{-}$-counterpart. Based on this data, a remineralization factor (RF) and demineralization (DF) factor benchmarked, respectively, to 1x SBF and the demineralization rate of human enamel are suggested as figures of merit of therapeutic performance of dental treatments. Taken together, the outcomes offer new insights that can inform clinicians and researchers on the selection of remineralization strategies.
\end{abstract}

Keywords: teeth, demineralization, remineralization, vertical scanning interferometry, fluoride toothpaste, CPP-ACP toothpaste

* Corresponding authors: B. Wu (benwu@ucla.edu) and G. Sant (gsant@ucla.edu) 
Revised for Submission to Dental Materials (2016)

\subsection{Introduction}

Remineralization and demineralization are dynamic processes that compete in oral environments. While caries is associated with bacterial activity in dental plaque [1,2], erosion involves intrinsic and extrinsic acidic substances [3-9]. Both conditions result from imbalances that favor tissue demineralization. Demineralized hydroxyapatite (HAP) surfaces are more susceptible to other damage, e.g., abrasion, and the combination of acid dissolution and mechanical stresses promotes further enamel removal by exposing underlying tissues to successive acid attack [1012]. As a result, there is significant interest in favoring remineralization and preventing demineralization. Besides enforcing dietary changes to reduce acidic food intake and hence demineralization [13], the routine use of topical agents can enhance remineralization [9,14-27].

Demineralization and/or remineralization of dental tissues has been widely studied using scanning electron microscopy [28], transmission electron microscopy [29], atomic force microscopy [30], indentation [31], microradiography [32], electron probe microanalysis [33], light induced fluorescence [34], secondary ion mass spectroscopy [7], confocal microscopy [35], electrochemical impedance spectroscopy [36], ultrasonic measurements [37], iodide permeability test [38] and contact profilometry [39]. However, microscopy techniques only offer qualitative 2D means for assessment [40]. Other techniques, e.g. microradiography and transmission electron microscopy are destructive and do not provide real-time evaluation. Further, the experimental conditions can also be limiting - e.g. in the case of microradiography the alignment and geometry of the X-ray beam can limit imaging precision at the sample edge [40]. While methods such as atomic force microscopy offer nanoscale resolution, the measurements are timeconsuming [7] and of limited statistical relevance, as only small lateral areas can be scanned in realistic durations.

Recently, non-contact profilometry (e.g., laser scanning or white light interferometry, WLI) has been proposed as a new method to study dental tissues [10,14-17,41-45]. By building on past studies, this paper establishes procedural details for full-field quantitative assessments of reactions (i.e. dissolution or precipitation) occurring on dental tissue surfaces in contact with a solution using vertical scanning interferometry (VSI). VSI allows quantifications of surface topographies with a vertical resolution of $\approx 0.1 \mathrm{~nm}$ and a lateral resolution of around $500 \mathrm{~nm}$. VSI uses interferometric objectives consisting of an objective lens, a reference mirror and a beamsplitter (see Figure 1). The most common interferometric objectives differ in terms of the mirror location, such that the mirror can be located between the objective lens and the beam-splitter (Mirau objective) or it can be placed in another position because of its larger size (a Michelson objective). In both cases, a source directs a light beam onto the sample surface through the interferometric objective, where the beam-splitter separates the light into two beams. One beam is reflected back by the reference mirror, while the other travels along the optical axis and interacts with the sample. This latter beam is reflected by the sample's surface. This results in an optical path difference between the two light beams and, a pattern of interference fringes forms when the beams are recombined.

This interference pattern is composed of light and dark bands: when the two beams are in phase their amplitudes are summed and a light band forms, whereas when the beams are out of phase their amplitudes are subtracted and a dark band of zero amplitude results. The interference fringes are sampled by a CCD (charge-coupled device) sensor and the signal is digitized and 
processed to obtain 3D topographical maps of the sample's surface. VSI offers distinct advantages over other techniques, including: (i) non-destructive evaluation, (ii) nanoscale resolution of surface profiles $(\approx 0.1 \mathrm{~nm}$ in the z-direction) with the ability to scan large lateral areas on the order of $10 \mathrm{~s}$ of $\mathrm{mm}^{2}$, in real time, and (iii) the ability to render statistically relevant quantifications of true reaction (dissolution or precipitation) rates, while duly accounting for the exposed (surface) area of the solid. Therefore, this paper uses VSI to quantify the dissolution rate of enamel and synthetic HAP at $\mathrm{pH} 4$. Further, the remineralizing effects of fluoride and CPPACP toothpastes are measured with subsequent analysis of demineralization behavior of the remineralized enamel surfaces.

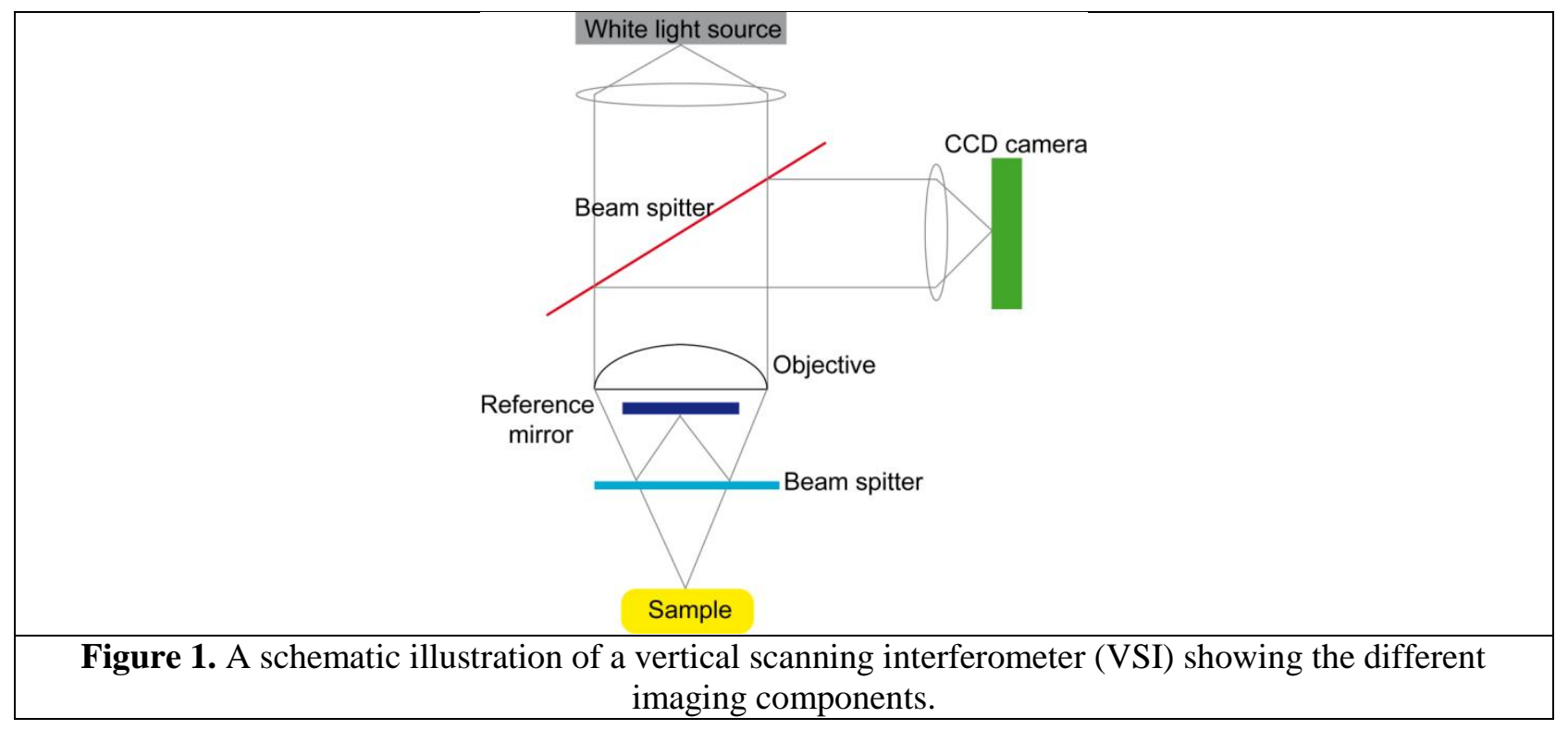

The first aim of this study is to establish VSI as a quantitative tool to evaluate demineralization and remineralization rates of dental tissues. The main motivation is to use real-time, continuous, high-resolution, quantitative data to rationalize the contradictory observations in the literature. For instance, Prabhakar et al. [19] reported fluoride-based toothpastes to be a superior remineralizing agent compared to CCP-ACP treatments, while Somasundaram et al. [20] noted opposite findings. Elsayad et al. [46] stated that fluoride-based and CCP-ACP toothpastes can have a "synergistic effect" on remineralization if used together; while Huang et al. and Balan et al. $[47,48]$ reported no significant difference in the effectiveness of these toothpastes. Besides minor differences in the treatment protocol, the studies also relied on analytical techniques that did not provide real time quantitative demineralization/remineralization rates. The second aim of this paper is to identify and benchmark therapeutic treatments that can mitigate acid attack of hard dental tissues.

\subsection{Material and methods}

\subsection{Sample preparation}

Caries-free, extracted human molars were collected from local oral surgery clinics. The teeth were sectioned axially using a precision slow speed diamond saw (IsoMet ${ }^{\mathrm{TM}} 1000$, Buehler Inc.). The sections comprised the buccal, lingual, mesial and distal walls of the tooth and had a size on the order of $5 \mathrm{~mm} \times 5 \mathrm{~mm} \times 5 \mathrm{~mm}$. After sectioning, the specimens were embedded in a cold- 
cured, inert, epoxy resin (EpoxiCure Resin, PN 203430128: Buehler Inc.), with the enamel portion facing outward and polished to a uniform smoothness using $\mathrm{SiC}$ abrasive sheets of grit levels ranging from 400-to-1200. The polished sections were fixed to a glass slide using an inert adhesive (double sided tape 3M 950, supplied by Uline) to facilitate handling. It should be noted that the samples are polished flat to remove natural variations in the surface of tooth sites that may result in different responses to acid attack $[49,50]$.

Powdered synthetic hydroxyapatite (HAP: $\mathrm{Ca}_{5}\left(\mathrm{PO}_{4}\right)_{3}(\mathrm{OH})$; molecular weight of $502.31 \mathrm{~g} / \mathrm{mole}$ ) was sourced from Acros Organics ${ }^{\mathrm{TM}}$ (CAS 1306-06-5). For dissolution analysis, powder particles with median diameter $\mathrm{d}_{50}=6.5 \mu \mathrm{m}$ were fixed on a glass slide using inert double sided tape (3M 950, supplied by Uline) prior to further analysis.

\subsection{De-/Re-mineralizating treatment protocol}

VSI was used to monitor the enamel surfaces over three steps: (Step 1) demineralization, (Step 2) remineralization, and (Step 3) demineralization of the newly remineralized surface (Figure 2).

Step 1: Demineralization is carried out at room temperature $\left(25^{\circ} \pm 3^{\circ} \mathrm{C}\right)$ using a $\mathrm{pH}=4$ buffer solution (Fisher Scientific CAS 50-00-0: water 98.91 \%, 1,2-Benzenedicarboxylic acid, monopotassium salt $1.0 \%$, formaldehyde $0.05 \%$, methyl alcohol $0.02 \%$, fluorescein, 2',4',5',7'-tetraiodo, disodium salt $0.02 \%$, all percentages written as mass \%). A few drops of buffer solution $(50-75 \mu \mathrm{L})$ were applied on the sample surface using a micropipette to obtain a liquid-to-solid ratio (1/s, by mass) between 50000-75000 to approximate the dilute limit. This elevated ratio hindered solution saturation with ions and prevents precipitation, if any. After 20 minutes of solvent contact, the sample is rinsed using distilled water and gently dried with compressed air. The dry sample is analyzed by VSI over fifteen cycle's demineralization cycles resulting in a solution contact time of 5 hours per sample. For comparison, the dissolution rate of synthetic hydroxyapatite (HAP) was also determined under the same experimental conditions.

Step 2: Enamel remineralization was carried out at room temperature using 2 commercial agents: (i) PreviDent5000 (Colgate-Palmolive, New York, USA) containing 1.1\% NaF, and (ii) MI Paste (GC Corporation, Tokyo, Japan) whose active ingredient is Recaldent ${ }^{\mathrm{TM}}$ (CPP-ACP: casein phosphopeptide, CPP, and amorphous calcium phosphate, ACP). Each toothpaste was applied on the enamel surface for a contact time of 60 minutes. This procedure was repeated 9 times (i.e., for a total application duration of 9 hours). Between each remineralization treatments, the samples were rinsed thoroughly and then dried prior to VSI analysis.

Besides comparing the toothpastes' remineralization efficacy against each other, it would be more beneficial to compare them to standard benchmark remineralization agents. Simulated body fluid (SBF) was selected as a benchmark agent because of its two advantages over artificial saliva:

(i) SBF is widely used and its composition (and ionic strength) can be closely controlled, and,

(ii) For the purposes of this study, i.e., to demonstrate the ability to detect varying degrees of mineralization, it is important to select an easily reproducible protocol that can be replicated by other investigators around the world.

SBF is a well-established agent (ISO 23317) that is suitable for use in international standardized protocols to evaluate biomineralization [52]. In contrast, it is difficult to replicate the unique 
properties of human saliva because this complex fluid is a mixture of fluids secreted by several salivary glands. Each of these individual saliva producers make highly variable saliva in terms of viscosity, ionic strength, etc. according to the time of day, diet, and many physiologic factors. For example, Gal. et al [53] investigated several different artificial saliva formulations and found that several were arbitrary formulations which yielded highly variable properties. While some of the more recent artificial saliva formulations contain similar ions as SBF, they do not allow simple additions of calcium and phosphates species to alter ionic strength, without producing insoluble precipitants. On the other hand, SBF is a well-designed system that allows easy manipulation of ionic strength without precipitation in most hands.

Therefore, three SBF concentrations (1x, 2x and 5x) were prepared, and their remineralization rates were used to provide data against which the commercial toothpastes could be compared. After demineralization at $\mathrm{pH} 4$ (Step 1), remineralization is carried out by contacting the enamel surface with 50-75 $\mu \mathrm{L}$ of SBF for 30 minutes. After each SBF application, the sample is rinsed with distilled water and dried with compressed air prior to VSI analysis. The remineralization step was repeated 14 times for a total contact time of 7 hours.

Step 3: Similar to Step 1, acid exposure $(\mathrm{pH}=4)$ was carried out to ascertain the demineralization rates of the "deposited or remineralized" tissue surfaces, as noted in Step 2.

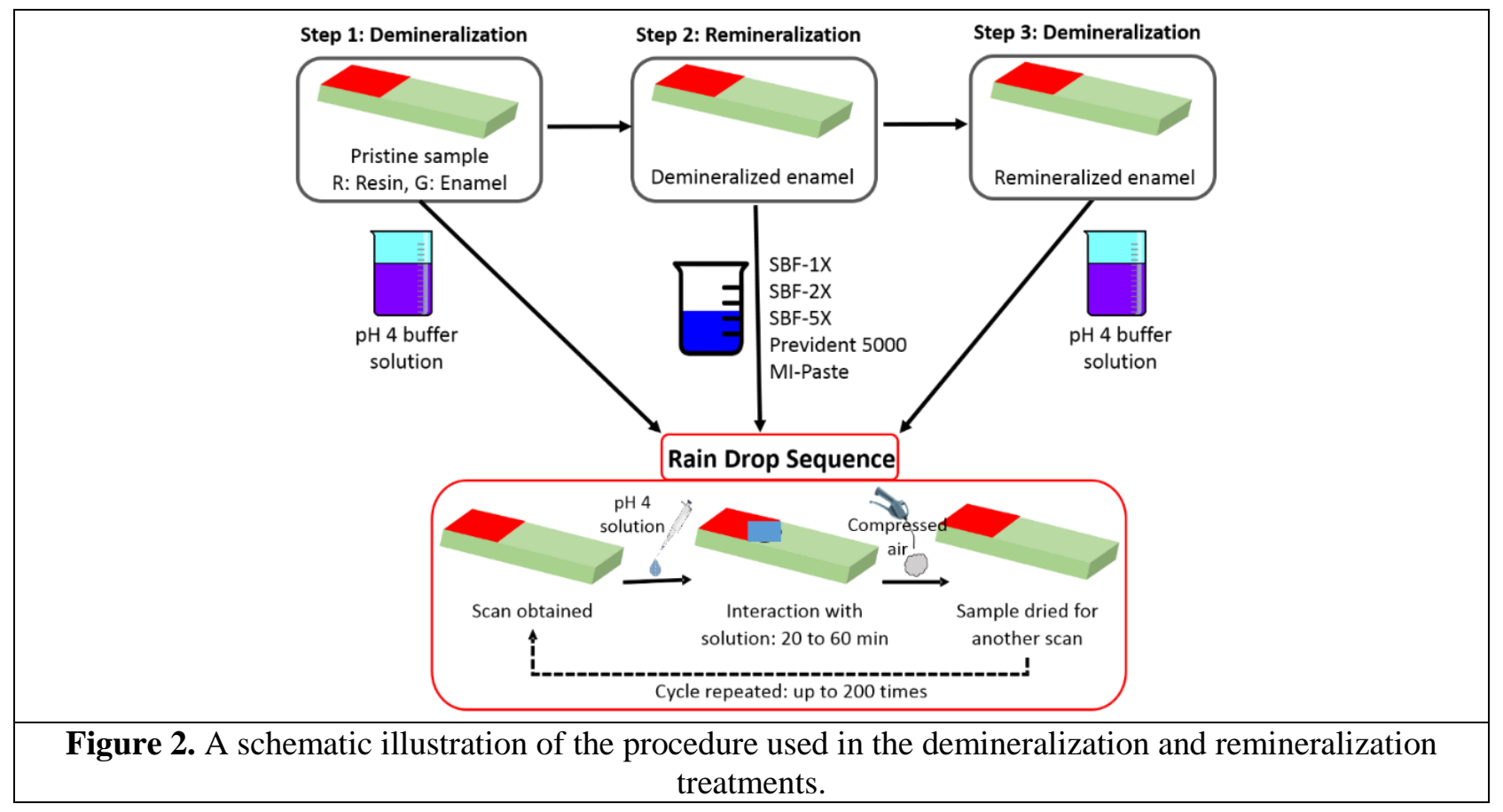

\subsection{Vertical Scanning Interferometry (VSI)}

A Zygo NV 8200 vertical scanning interferometer fitted with a 50x Mirau objective (N.A. = 0.55) was used in the analyses. This setup permitted visualization of a scanning area of 152.27 $\mu \mathrm{m} \times 141.86 \mu \mathrm{m}$ per image field. Since images are recorded in stitched mode, using a P x Q grid ( $\mathrm{P}$ rows $\mathrm{x} \mathrm{Q}$ columns) composed of $\mathrm{PQ}$ tiles, the full-image field is on the order of $(152.27 \cdot \mathrm{P})$ $\mu \mathrm{m} \times(141.86 \cdot \mathrm{Q}) \mu \mathrm{m}$ wherein $\mathrm{P}$ and $\mathrm{Q}$ values ranged between 2 to 5 . A back-scan length of 145 $\mu \mathrm{m}$ was used, which allows peak-to-valley height differences up to $290 \mu \mathrm{m}$ to be sampled. The 
acquisition time of the tiled image field, depending on grid size, ranged between 300-to-1200 seconds.

For any given sample, a scanning area was selected to monitor the topography of the surface (i.e., enamel or HAP), while establishing the embedment resin (for the enamel) or the polymer substrate (for the HAP), as a reference plane. Both the resin and the polymer used are inert to the solutions applied (see Section 3.1). After the images are captured, a data processing procedure consisting of several steps is applied:

(i) filtering is carried out to include or exclude specific characteristics of the surface. For the data presented herein, a median filter is chosen to reduce noise.

(ii) Form removal is carried out, if needed, to minimize alignment errors and to remove any background tilt that could mask details of the surface. To perform such leveling, mathematical functions are chosen to describe a geometrical form (e.g., a plane) that can be fit to, and then subtracted from the acquired data.

(iii) Masking is used to select and analyze a specific region in detail, while excluding (masking) the rest of the image field from the analyses. With the use of multiple masks placed over different regions of a given image-field, topographical parameters spanning a large area can be acquired, and, as such, a statistically significant analysis can be performed.

(iv) Topographical parameters are calculated using the Mx software (Ver. 6.1.0.4, distributed by Zygo Corporation) to quantify the changes in surface height. The parameter used in this study is the area averaged height $(h)$ that is computed by averaging the height profile over $M \times N$ pixels as follows [54]:

$$
h=\frac{1}{M N} \sum_{x=0}^{M-1} \sum_{y=0}^{N-1} Z(x, y)-\bar{Z}
$$

where, $M$ and $N$ are the number of pixels along the $x$ and $y$ directions, $Z$ is the height of the sample $(\mathrm{nm})$ at the location of the pixel located at $(x, y)$, and $\bar{Z}$ is the height of the reference plane (i.e., XY plane with respect to fixed origin in 3D space; in this case the epoxy in which the enamel is embedded). The difference between $\mathrm{Z}$ and $\bar{Z}$, therefore, is a measure of the height of a given feature on the sample with respect to the reference plane.

(v) The demineralization or remineralization rates $\left(D_{R}\right.$, or $\left.R_{R}\right)$ is computed as:

$$
\mathrm{D}_{R}(\mathrm{~m} / \mathrm{s})=\Delta h / \Delta t
$$

where, $\Delta h=h(i)-h(i+1)$ is the change in the average height $(\mathrm{nm})$ of the exposed material with respect to the reference plane between step $(i)$ and its successive step $(i+1)$ measured over a period of time $\Delta t$ (seconds). The highest uncertainty in dissolution and precipitation rates, quantified in this manner is on the order of 10\% [54-56]. The reproducibility of the experimental results is examined by repeating each experiment three times under the same conditions.

\subsection{Scanning Electron Microscopy (SEM)}

SEM images of select enamel samples were obtained before and after demineralization and after subsequent remineralization. Imaging was carried out using secondary electrons (SE) using a FEI Nova NanoSEM $230(10 \mathrm{kV}, 80 \mathrm{pA})$. The images allow for qualitative comparison of the pristine 
enamel surfaces before the application of any treatment, and following demineralization (Step 1, $\mathrm{t}=5 \mathrm{~h}$ ) and remineralization (Step 2, $\mathrm{t}=14 \mathrm{~h}$ ).

\subsection{X-Ray Diffraction (XRD)}

XRD patterns were obtained on demineralized enamel surfaces treated with a $\mathrm{F}^{-}$-based and CPPACP toothpastes using a Bruker D8 diffractometer with $\theta-\theta$ "Bragg-Brentano" geometry, using $\mathrm{Cu}-\mathrm{K} \alpha$ radiation $(\lambda=1.5406 \AA), 40 \mathrm{kV}$ accelerating voltage, and $40 \mathrm{~mA}$ beam current. The samples were scanned on a rotating stage over $5^{\circ}-75^{\circ} 2 \theta$ range with a scan step of $0.02^{\circ}(2 \theta)$ and a step time of exposure time of 0.15 second per step.

\section{Results and discussion}

\subsection{Method of analysis}

To quantify the dissolution (demineralization) or precipitation (remineralization) rates, the height of the enamel surface was measured. During demineralization treatments (Step 1 and 3) the height progressively decreases, whereas during remineralization treatment (Step 2) the height increases (Figure 3). The change in height of the surface are calculated relative to the height of the reference plane $\bar{Z}$ (Eq. 1), which remains fixed throughout the experiment. The stability of the reference plane (i.e., epoxy resin) was confirmed by several experiments, wherein after prolonged exposure to acidic or basic solutions (for $4 \leq \mathrm{pH} \leq 12$ ), its change in height (i.e., of the resin) was found to be less than $0.1 \mathrm{~nm}$; which is within the measurement's resolution [54-56]. It should also be noted that additional pilot experiments carried out which involved the submersion of enamel and resin in water confirmed that no volume changes occurred; following gentle drying for $\leq 1$ minute.

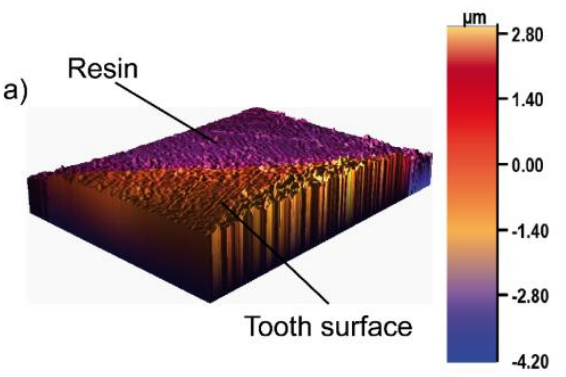

b)
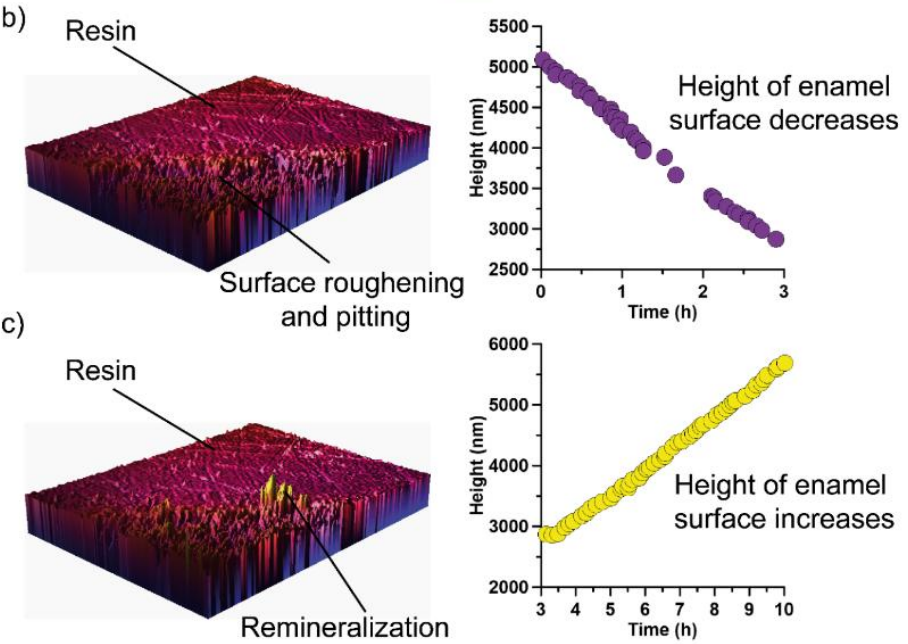

Figure 3. Representative topographical maps obtained from the VSI analysis. The isometric images show 
the pristine, demineralized and remineralized enamel sections. (a) The top-left corner represents the resin reference, whereas the remainder shows the exposed enamel surface. (b) Shows a retreat in the spatially averaged height of the enamel surface (due to valleys and pits formed on the enamel surface; bottom-right corner of image), with respect to the resin reference due to demineralization. (c) Shows the formation of precipitates (see re-emergent peaks of material) following remineralization of the enamel surface. Each data point shown features an uncertainty of $\pm 14 \%$.

The demineralization or remineralization rates are computed using Eq. (2) by averaging over different regions within a larger scanning area to obtain a statistically relevant value, which duly accounts for both exposed surface area and roughness. Therefore, the values of $D_{R}$ (dissolution rate, $\mathrm{nm} / \mathrm{h}$ ) and $\mathrm{R}_{\mathrm{R}}$ (remineralization rate, $\mathrm{nm} / \mathrm{h}$ ) are independent of the presence or the absence of heterogeneities which are randomly distributed across the surface.

\subsection{Dissolution of enamel and synthetic hydroxyapatite}

The dissolution rate of enamel was compared with that of hydroxyapatite (HAP), because HAP is a dominant inorganic constituent of teeth. The dissolution of HAP under acidic conditions has been studied to understand the evolution of pathologies such as osteoporosis, dental caries and erosion. Nevertheless, the dissolution of hydroxyapatite has not been fully clarified, and even now, several models and competing mechanisms continue to be proposed to describe its progress [57] including: (i) diffusion control, (ii) surface control, (iii) polynuclear model, (iv) selfinhibition, (v) congruent or incongruent dissolution; (vi) chemical control; (vii) etch pit formation, (viii) ion exchange and (ix) the hydrogen catalytic model.

The dissolution rate of synthetic HAP at low $\mathrm{pH}(\mathrm{pH} \leq 4)$ was previously compared to that of enamel $[58,59]$ indicating that the latter is faster dissolving than the former. But our results suggest that enamel dissolves slower than HAP at $\mathrm{pH} 4$ (Figure 4). This difference is thought to arise because dissolution rates can be affected by chemical substitutions in the HAP crystallite structure of dental enamel. For example, it is well-known that fluoride substitutions reduce the dissolution rate of HAP (i.e., in enamel) while the inclusion of carbonate ions destabilizes the HAP structure, thus, increasing its tendency to dissolve [60,61]. Significant fluoride ion uptake in the enamel, e.g., resulting from the use of fluoridated treatments can result in suppressing enamel dissolution rates vis-à-vis synthetic (pure) HAP.

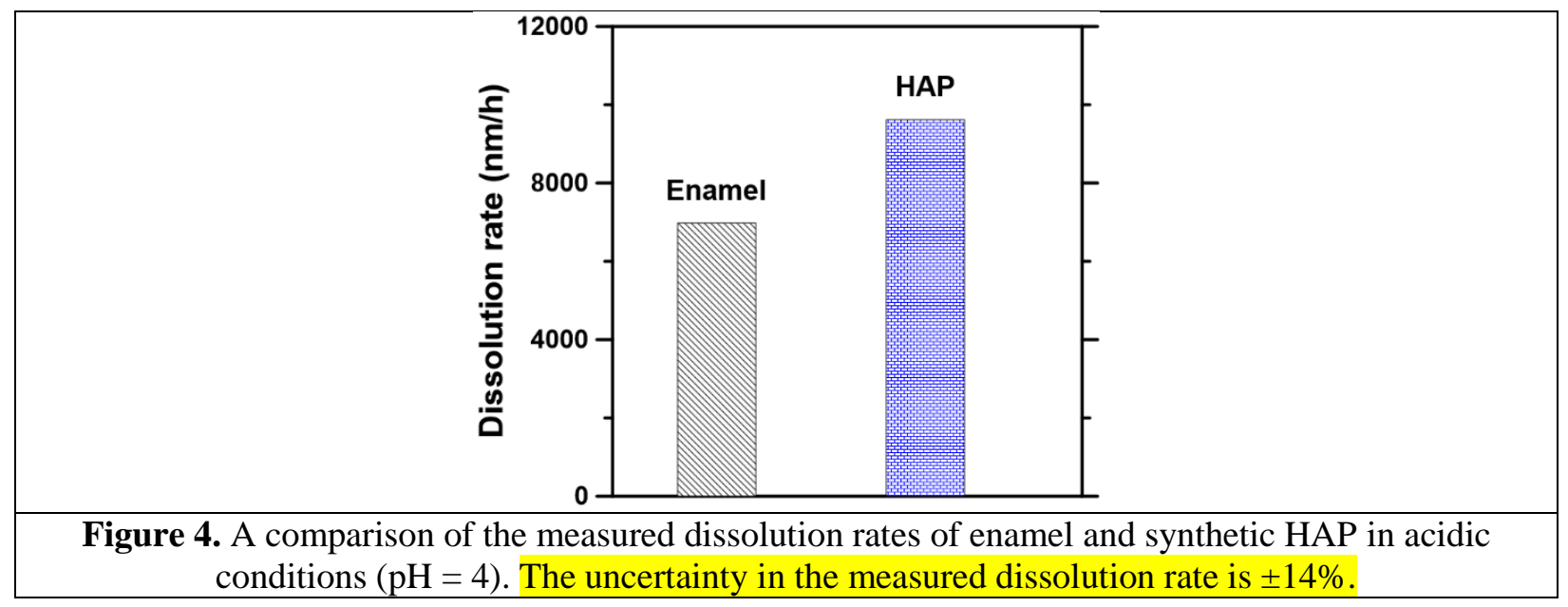


It should be noted that while the VSI technique can analyze erosive surface lesions, it cannot detect subsurface changes which can be produced by cyclic demineralizing regime. However, the type of lesion formed depends on the cause of destruction. Dietary acids and bases can induce surface erosion, as can many combinations of bacteria in dental plaque. For example, while Streptococcus mutans alone, and the combination of S. mutans and Lactobacillus acidophilus tend to induce subsurface lesions, the combination of S. mutans and L. casei, or S. mutans, L. acidophilus, and L. casei, has been shown to produce surface cavitations [51]. Nevertheless, the application of VSi for analysis of surface erosion is relevant, independent of the cause/origin of such erosion.

\subsection{Remineralization treatments}

Previous studies on the effectiveness of topical dental therapies lack consensus. Several studies have suggested that fluoride toothpastes reduce demineralization and enhance remineralization $[14,17,24]$. Others claimed that fluoride treatments do not prevent demineralization [62] and that intensive fluoridation can cause oral mucosa irritation [63]. On the other hand, the application of CCP-ACP toothpastes is recommended elsewhere [20,50,64], although the effectiveness of both fluoride and CCP-ACP-based treatments has been questioned by Huang et al. [47], who noted no differences following their application of these treatments vis-à-vis standard oral hygiene.

To resolve these contradictions, the rates of remineralization of a variety of treatments were quantified using a consistent protocol. The rates of remineralization were quantified using SBF (simulated body fluid; [52]) prepared at different strengths (concentrations) as a benchmark (see Figure 5). It was noted that the SBF remineralization rates follow a natural log function in relation to strength. When compared to the SBF treatments, it is noted that PreviDent5000 and the MI Paste induce remineralization at rates that would correspond to SBF strengths of 4.6x and 1.7x respectively. Using the remineralization rate of SBF $1 \mathrm{x}$ as a reference, the efficacy of MI Paste and PreviDent5000 were benchmarked by a remineralization factor (RF, unitless) noted in Eq. (3).

\begin{tabular}{|l|l|}
\hline $\mathbf{R F}=\frac{\text { Remineralization rate of toothpaste }}{\text { Remineralization of SBF }(1 \mathrm{x})}$ & (3) \\
\hline
\end{tabular}

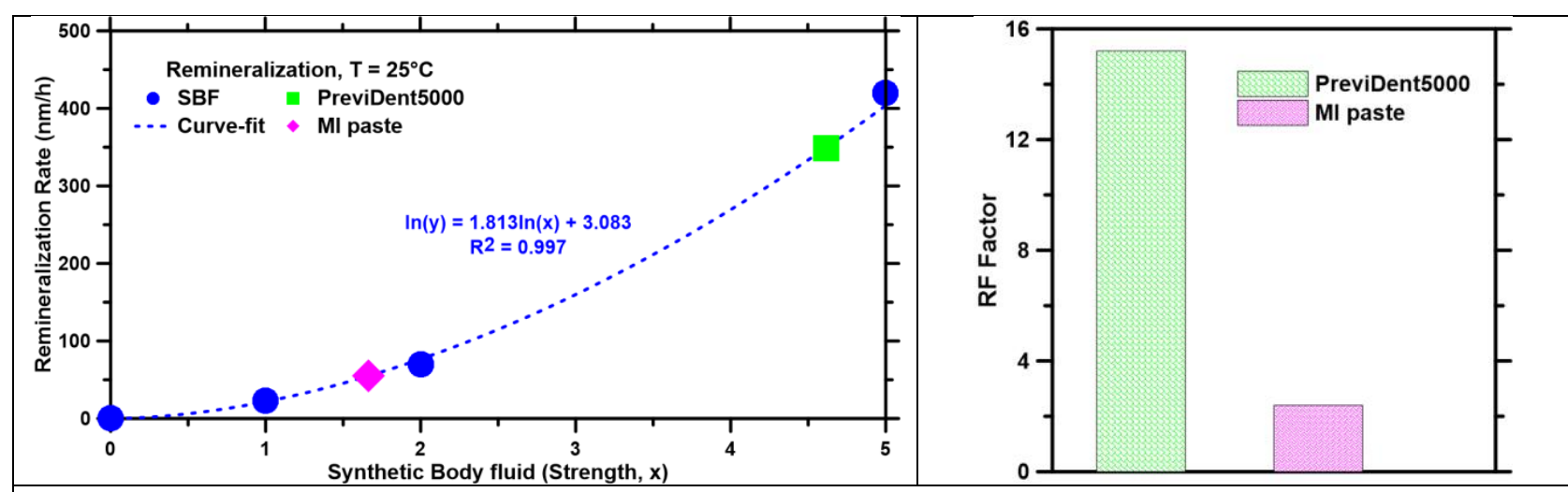

Figure 5. (a) The remineralization rates of enamel surfaces following their exposure to SBF prepared at three strengths (1x, 2x, 5x; circles) and two commercially available treatments: a fluoride toothpaste (PreviDent5000; square) and a CPP-ACP toothpaste (MI Paste; diamond). By using SBF remineralization rates as a reference, a method is established to compare quantitative remineralization rates of other non- 
SBF treatment. (b) The remineralization factors (RF) of the two toothpastes. The uncertainty in the remineralization rates is $\pm 14 \%$.

Such analysis revealed that PreviDent5000 has a higher remineralizing capability, with $\mathrm{RF}=$ 15.2 , as compared to MI Paste, which yields $\mathrm{RF}=2.4$. While this may explain the outcomes of previous studies that suggest that fluoride-based toothpastes are superior remineralizing agents $[17,19,65-68]$, it should be emphasized that RF is only a kinetic indicator of remineralization, and it does not predict the stability of the deposited precipitates, which can be determined by subjecting this new precipitant to dissolution. The remineralization effect of fluoride-based treatments is attributed to the rapid formation of $\mathrm{NaF}$ precipitates, that protect enamel surfaces

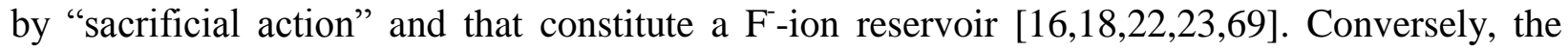
remineralization kinetics of MI Paste are known to be lower than other treatments $[19,50,65]$, in agreement with our results.

\subsection{Dissolution of new precipitants (stability of remineralized surface)}

To determine the stability of the remineralized surface against acid attack, a second sequence of demineralization is carried out to assess the stability of the newly formed precipitant. In this case, the rate of demineralization is quantified by a demineralization factor (DF, unitless) which describes the kinetic resistance to dissolution, of the precipitated phases, vis-à-vis pristine dental enamel, where:

$$
\mathrm{DF}=\frac{\text { Demineralization rate after toothpaste treatment }}{\text { Demineralization rate of untreated enamel }}
$$

Figure 6 shows that the $\mathrm{F}^{-}$treated sample demineralized faster $(\mathrm{DF}=1.6)$ than the pristine enamel, whereas the CPP-ACP paste treated sample demineralized slower than pristine enamel $(\mathrm{DF}=0.6)$. This difference can be explained by the fact that the $\mathrm{NaF}$ bearing deposits (i.e., identified by X-ray diffraction, Figure 7) that formed during PreviDent5000 application, served as a sacrificial material that "preferentially dissolves" before the underlying enamel is attacked by acid [23]. This sacrificial behavior is likely on the account of the soluble nature of these deposits, which dissolve faster than crystalline HAP that constitutes dental tissues. It is also important to highlight that the XRD pattern (Figure 7) shows low intensity peaks at $47^{\circ} 2 \Theta$ and $28.3^{\circ} 2 \Theta$, which suggests the presence of $\mathrm{CaF}_{2}$. However, given the much larger intensities of the principal $\mathrm{NaF}$ peaks vis-à-vis $\mathrm{CaF}_{2}$ peaks; $\mathrm{NaF}$ exists as the dominant precipitate formed.

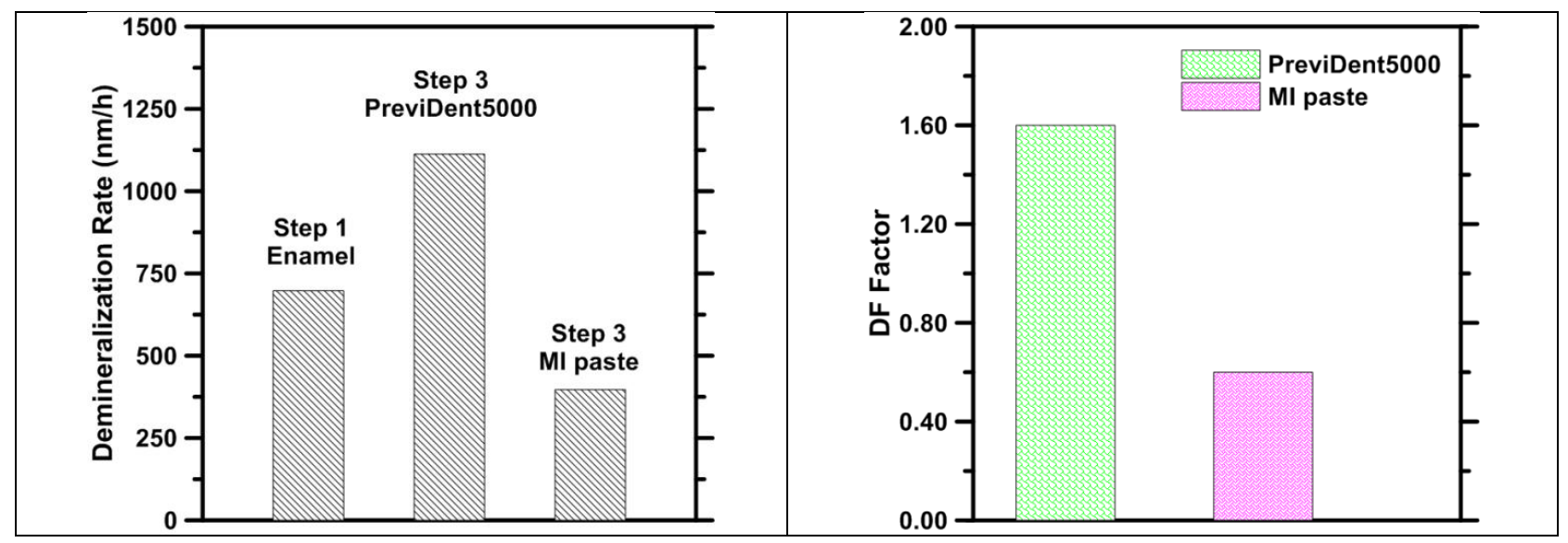


(a)

(b)

Figure 6. (a) A comparison of the demineralization rates of pristine enamel (Step 1), and the demineralization rates of previously demineralized enamel surfaces that are treated with a fluoride-based and CPP-ACP toothpaste (Step 3). (b) The demineralization factors (DF) of both commercial toothpastes.

It should be noted that the $\mathrm{NaF}$ deposits formed on $\mathrm{F}^{-}$treated samples appear globular (2000x, see Figure 8d). On the contrary, the SEM images of pristine teeth showed elongated hydroxyapatite crystals within the typical honeycomb structure of teeth (Figures 8b, c). The instability of the $\mathrm{NaF}$ deposits was also noted by Hove et al. [15], who reported that these deposits are likely effective only for a limited period, and while they may provide resistance against acid attack initially, this resistance declined progressively after repeated and prolonged acid exposure - as the $\mathrm{NaF}$ deposits are progressively removed. The rapid dissolution of the $\mathrm{NaF}$ deposits at low pHs is also confirmed by $[48,62]$ - an explanation of why fluoride-based toothpastes do not prevent dental erosion at longer time scales; unless repeated, and regular toothpaste applications are carried out.

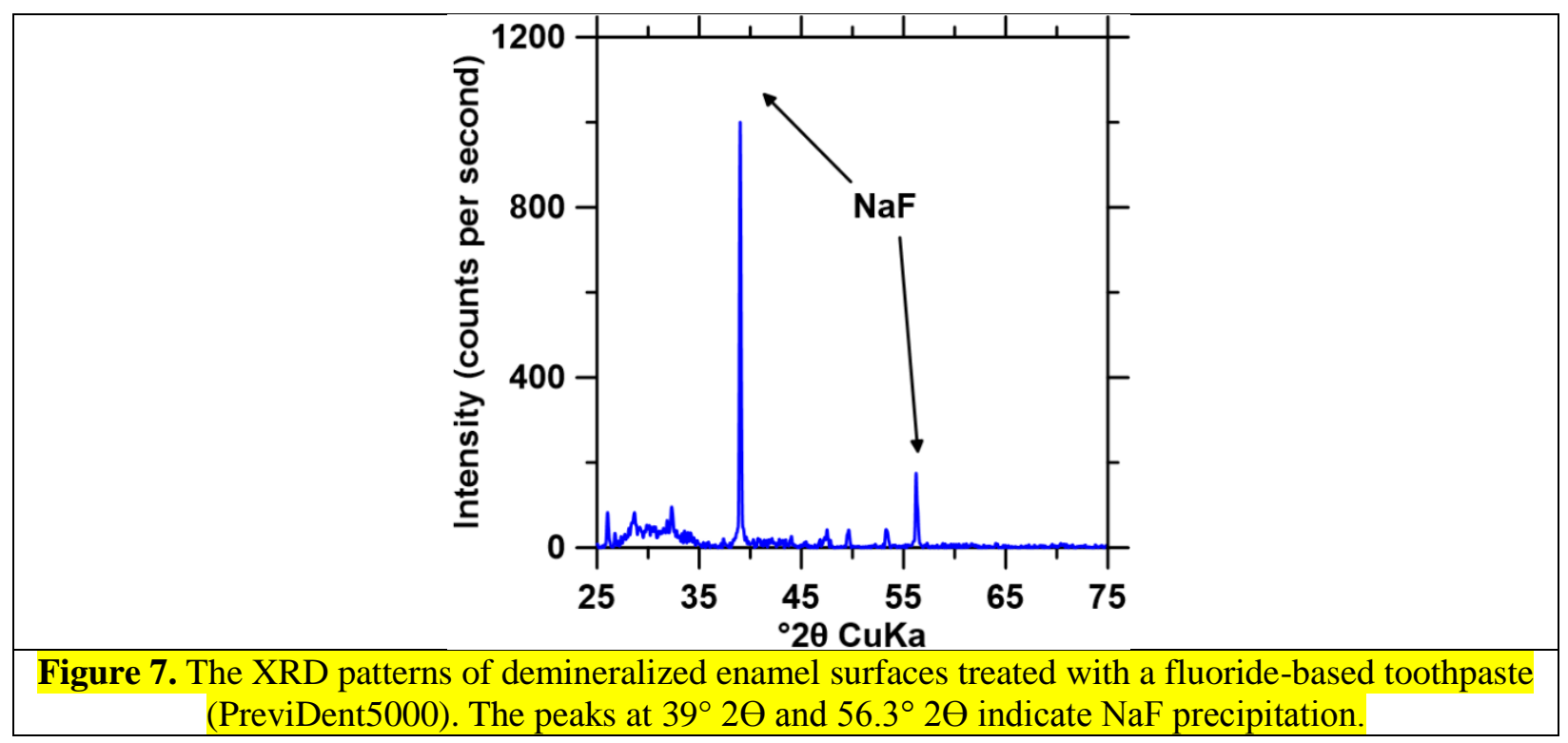

The precipitate formed during remineralization by the CPP-ACP toothpaste is far more resistant (from a kinetic perspective) to acid attack than even pristine enamel [20,50,70] (Figure 6). Thus, even if the CPP-ACP toothpaste did not result in significant remineralization (in terms of rate and hence the thickness of the precipitated layer), the deposited material provided excellent protection against dental erosion. This protective action is a result of CPP-ACP's behavior which increased the number of $\mathrm{Ca}^{2+}$-binding sites, decreased diffusion, and reduced calcium loss (i.e., by leaching) from enamel during acid exposure [71,72]. Casein phosphopeptide (CCP) is also thought to stabilize amorphous calcium phosphate (ACP) on enamel surfaces, thereby buffering calcium and phosphate ion activities, preserving their supersaturation, i.e., in the proximity of enamel, thereby retarding the latter's demineralization [50,73,74]. Zhang et al. [75] also attributed the better acid resistance of the $\mathrm{CCP}-\mathrm{ACP}$ remineralization product to the formation of nano-size particulates which are homogenously arranged and more tightly packed, than those formed by the application of fluoride toothpaste. This resulted also in the formation of physical barriers in the porous zones of enamel making it more resistant to future demineralization. 
Taken together, the discussion above suggests that the application of Prevident5000 is better suited when prescribed for regular and repeated application, over shorter durations. On the other hand, MI Paste is likely a better treatment in the long term, but an extended duration of application may be needed (e.g., using a dental tray) for substantial benefit to be noted. VSI reveals the quantitative differences in the behavior and performance of fluoride and CPP-ACP toothpastes, and highlights the relative strengths and weaknesses for experts in the field to debate. Moreover, the results also suggest that the application of NaF + CPP-ACP treatments may be synergistic - because while the former rapidly forms a precipitate, the latter forms a protective layer that envelopes the otherwise readily-soluble $\mathrm{NaF}$ deposit - as a result, "synergistic" benefits are expected.

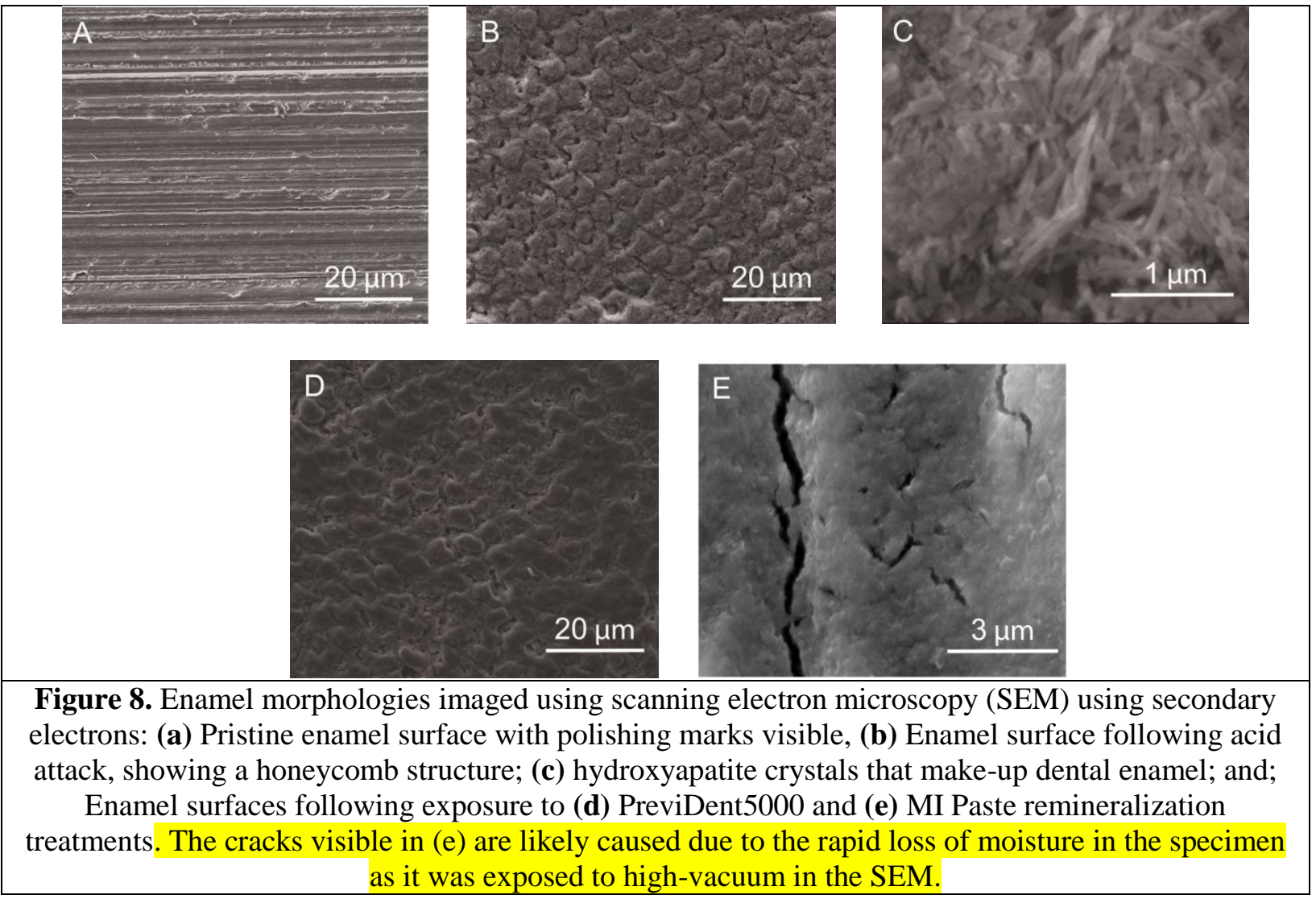

\subsection{Conclusions}

This study demonstrates the application of vertical scanning interferometry (VSI) as a quantitative, rapid and high-resolution methodology to study solid-solution reactions of dental tissues. The nanoscale resolution of the VSI method is exploited to study the kinetics of both, demineralization and remineralization of dental enamel. Special focus is placed on quantifying the kinetics of these processes and the effectiveness of commercially available remineralization treatments. The VSI quantification form a basis to propose a demineralization (DF) and remineralization (RF) factor as figures of merit to compare quantitatively, the performance of two remineralizing agents, e.g. PreviDent5000 and MI Paste. Analysis of these factors and imaging of the hard tissue surfaces allowed for better understanding of how these treatments 
work, and resolution of inconsistences previously noted in their healing performance. The results unambiguously indicate that while PreviDent5000 offers fast remineralization rates $(\approx 6.3 \mathrm{x}$ higher than MI Paste), the precipitates formed following its application dissolved 2.7x times faster than those formed by MI Paste.

\section{Acknowledgements}

The authors acknowledge financial support for this research provided by in part by: The Oak Ridge National Laboratory operated for the U.S. Department of Energy by UT-Battelle (LDRD Award \# 4000132990), the National Science Foundation (ENG-CMMI: 1401533 and CAREER: 1235269), Federal Highway Administration (DTFH61-13-H-00011) and the University of California, Los Angeles (UCLA). The contents of this paper reflect the views and opinions of the authors, who are responsible for the accuracy of data presented herein. This research was conducted in: Laboratory for the Chemistry of Construction Materials $\left(\mathrm{LC}^{2}\right)$, ElectronMicroscopy Core, and, Molecular Instrumentation Center (MIC) at UCLA. The authors gratefully acknowledge the financial support that has made these laboratories and their operations possible.

\section{References}

[1] W.J. Loesche, Role of Streptococcus mutans in human dental decay, Microbiol. Rev. 50 (1986) 353-380.

[2] I. Kleinberg, A Mixed-bacteria Ecological Approach to Understanding the Role of the Oral Bacteria in Dental Caries Causation: an Alternative to Streptococcus mutans and the Specific-plaque Hypothesis, Crit. Rev. Oral Biol. Med. 13 (2002) 108-125. doi: $10.1177 / 154411130201300202$.

[3] T. Imfeld, Dental erosion. Definition, classification and links, Eur. J. Oral Sci. 104 (1996) 151-155. doi:10.1111/j.1600-0722.1996.tb00063.x.

[4] C. Asher, M.J. Read, Early enamel erosion in children associated with the excessive consumption of citric acid, Br. Dent. J. 162 (1987) 384-387.

[5] V.K. Jarvinen, I.I. Rytomaa, O.P. Heinonen, Risk factors in dental erosion, J. Dent. Res. 70 (1991) 942-947.

[6] D.T. Zero, Etiology of dental erosion - extrinsic factors, Eur. J. Oral Sci. 104 (1996) 162177. doi:10.1111/j.1600-0722.1996.tb00065.x.

[7] M.E. Barbour, J.S. Rees, The laboratory assessment of enamel erosion: a review, J. Dent. 32 (2004) 591-602. doi:10.1016/j.jdent.2004.05.001.

[8] M.E. Barbour, R.P. Shellis, An investigation using atomic force microscopy nanoindentation of dental enamel demineralization as a function of undissociated acid concentration and differential buffer capacity, Phys. Med. Biol. 52 (2007) 899-910. doi:10.1088/0031-9155/52/4/003.

[9] Y.-F. Ren, Q. Zhao, H. Malmstrom, V. Barnes, T. Xu, Assessing fluoride treatment and resistance of dental enamel to soft drink erosion in vitro: Applications of focus variation 3D scanning microscopy and stylus profilometry, J. Dent. 37 (2009) 167-176. doi:10.1016/j.jdent.2008.09.008.

[10] T. Attin, S. Knöfel, W. Buchalla, R. Tütüncü, In situ evaluation of different remineralization periods to decrease brushing abrasion of demineralized enamel, Caries Res. 35 (2001) 216-222. doi:47459. 
[11] M. Eisenburger, R.P. Shellis, M. Addy, Comparative study of wear of enamel induced by alternating and simultaneous combinations of abrasion and erosion in vitro, Caries Res. 37 (2003) 450-455. doi:73399.

[12] E. Heurich, M. Beyer, K.D. Jandt, J. Reichert, V. Herold, M. Schnabelrauch, B.W. Sigusch, Quantification of dental erosion-A comparison of stylus profilometry and confocal laser scanning microscopy (CLSM), Dent. Mater. $26 \quad$ (2010) 326-336. doi:10.1016/j.dental.2009.12.001.

[13] J.F. Tahmassebi, M.S. Duggal, G. Malik-Kotru, M.E.J. Curzon, Soft drinks and dental health: a review of the current literature, J. Dent. 34 (2006) 2-11. doi:10.1016/j.jdent.2004.11.006.

[14] L. Hove, B. Holme, B. Øgaard, T. Willumsen, A.B. Tveit, The protective effect of $\mathrm{TiF}_{4}$, $\mathrm{SnF}_{2}$ and $\mathrm{NaF}$ on erosion of enamel by hydrochloric acid in vitro measured by white light interferometry, Caries Res. 40 (2006) 440-443. doi:10.1159/000094291.

[15] L.H. Hove, B. Holme, A. Young, A.B. Tveit, The erosion-inhibiting effect of $\mathrm{TiF}_{4}, \mathrm{SnF}_{2}$, and $\mathrm{NaF}$ solutions on pellicle-covered enamel in vitro, Acta Odontol. Scand. 65 (2007) 259-264. doi:10.1080/00016350701477581.

[16] L.H. Hove, B. Holme, A. Young, A.B. Tveit, The Protective Effect of $\mathrm{TiF}_{4}, \mathrm{SnF}_{2}$ and $\mathrm{NaF}$ against Erosion-Like Lesions in situ, Caries Res. 42 (2008) 68-72. doi:10.1159/000112816.

[17] S.E. Cross, J. Kreth, R.P. Wali, R. Sullivan, W. Shi, J.K. Gimzewski, Evaluation of bacteria-induced enamel demineralization using optical profilometry, Dent. Mater. 25 (2009) 1517-1526. doi:10.1016/j.dental.2009.07.012.

[18] M.D. Lagerweij, W. Buchalla, S. Kohnke, K. Becker, À. M. Lennon, T. Attin, Prevention of Erosion and Abrasion by a High Fluoride Concentration Gel Applied at High Frequencies, Caries Res. 40 (2006) 148-153. doi:10.1159/000091062.

[19] A.R. Prabhakar, A.J. Manojkumar, N. Basappa, In vitro remineralization of enamel subsurface lesions and assessment of dentine tubule occlusion from $\mathrm{NaF}$ dentifrices with and without calcium, J. Indian Soc. Pedod. Prev. Dent. 31 (2013) 29-35. doi:10.4103/09704388.112403 .

[20] P. Somasundaram, N. Vimala, L.G. Mandke, Protective potential of casein phosphopeptide amorphous calcium phosphate containing paste on enamel surfaces, J. Conserv. Dent. JCD. 16 (2013) 152-156. doi:10.4103/0972-0707.108199.

[21] C.C. De Souza, J.L.M. Cury, T.C.L. Coutinho, E.M. Da Silva, M.A. Tostes, Effect of different application frequencies of CPP-ACP and fluoride dentifrice on demineralized enamel: a laboratory study, Am. J. Dent. 27 (2014) 215-219.

[22] C. Ganss, J. Klimek, V. Brune, A. Schürmann, Effects of two fluoridation measures on erosion progression in human enamel and dentine in situ, Caries Res. 38 (2004) 561-566. doi: $10.1159 / 000080587$.

[23] X. Wang, B. Megert, E. Hellwig, K.W. Neuhaus, A. Lussi, Preventing erosion with novel agents, J. Dent. 39 (2011) 163-170. doi:10.1016/j.jdent.2010.11.007.

[24] R.V. Faller, S.L. Eversole, G.E. Tzeghai, Enamel protection: a comparison of marketed dentifrice performance against dental erosion, Am. J. Dent. 24 (2011) 205-210.

[25] R.S. Austin, J.M. Rodriguez, S. Dunne, R. Moazzez, D.W. Bartlett, The effect of increasing sodium fluoride concentrations on erosion and attrition of enamel and dentine in vitro, J. Dent. 38 (2010) 782-787. doi:10.1016/j.jdent.2010.06.009. 
[26] C.-C. Hsu, H.-Y. Chung, E.M. Hagerman, W. Shi, J.-M. Yang, B. Wu, Effects on Hardness and Elastic Modulus for DSS-8 Peptide Treatment on Remineralization of Human Dental Tissues, MRS Proc. 1132 (2008). doi:10.1557/PROC-1132-Z09-05.

[27] D.K. Yarbrough, E. Hagerman, R. Eckert, J. He, H. Choi, N. Cao, K. Le, J. Hedger, F. Qi, M. Anderson, B. Rutherford, B. Wu, S. Tetradis, W. Shi, Specific Binding and Mineralization of Calcified Surfaces by Small Peptides, Calcif. Tissue Int. 86 (2010) 58-66. doi:10.1007/s00223-009-9312-0.

[28] J.H. Meurman, R.M. Frank, Scanning Electron Microscopic Study of the Effect of Salivary Pellicle on Enamel Erosion, Caries Res. 25 (1991) 1-6. doi:10.1159/000261335.

[29] T. Yanagisawa, Y. Miake, High-resolution electron microscopy of enamel-crystal demineralization and remineralization in carious lesions, J. Electron Microsc. (Tokyo). 52 (2003) 605-613.

[30] F. Watari, In situ quantitative analysis of etching process of human teeth by atomic force microscopy, J. Electron Microsc. (Tokyo). 54 (2005) 299-308. doi:10.1093/jmicro/dfi056.

[31] H. Devlin, M.A. Bassiouny, D. Boston, Hardness of enamel exposed to Coca-Cola and artificial saliva, J. Oral Rehabil. 33 (2006) 26-30. doi:10.1111/j.1365-2842.2006.01533.x.

[32] B. Ogaard, H. Duschner, J. Ruben, J. Arends, Microradiography and confocal laser scanning microscopy applied to enamel lesions formed in vivo with and without fluoride varnish treatment, Eur. J. Oral Sci. 104 (1996) 378-383.

[33] B. Willershausen, B. Schulz-Dobrick, In vitro study on dental erosion provoked by various beverages using electron probe microanalysis, Eur. J. Med. Res. 9 (2004) 432-438.

[34] F. Lippert, A. Butler, R.J.M. Lynch, Enamel demineralization and remineralization under plaque fluid-like conditions: a quantitative light-induced fluorescence study, Caries Res. 45 (2011) 155-161. doi:10.1159/000325743.

[35] A.M.A. Maia, C. Longbottom, A.S.L. Gomes, J.M. Girkin, Enamel erosion and prevention efficacy characterized by confocal laser scanning microscope, Microsc. Res. Tech. 77 (2014) 439-445. doi:10.1002/jemt.22364.

[36] Y.-M. Liao, Z.-D. Feng, Z.-L. Chen, In situ tracing the process of human enamel demineralization by electrochemical impedance spectroscopy (EIS), J. Dent. 35 (2007) 425-430. doi:10.1016/j.jdent.2006.11.011.

[37] M.C. Huysmans, J.M. Thijssen, Ultrasonic measurement of enamel thickness: a tool for monitoring dental erosion?, J. Dent. 28 (2000) 187-191.

[38] F. Brudevold, A. Tehrani, R. Cruz, The Relationship Among the Permeability to Iodide, Pore Volume, and Intraoral Mineralization of Abraded Enamel, J. Dent. Res. 61 (1982) 645-648. doi:10.1177/00220345820610050501.

[39] S.M. Hooper, J.A. Hughes, R.G. Newcombe, M. Addy, N.X. West, A methodology for testing the erosive potential of sports drinks, J. Dent. 33 (2005) 343-348. doi:10.1016/j.jdent.2004.10.002.

[40] T. Attin, Methods for assessment of dental erosion, Monogr. Oral Sci. 20 (2006) 152-172. doi:10.1159/000093361.

[41] C.A. Hemingway, D.M. Parker, M. Addy, M.E. Barbour, Erosion of enamel by noncarbonated soft drinks with and without tooth brushing abrasion, Br. Dent. J. 201 (2006) 447-450; discussion 439; quiz 466. doi:10.1038/sj.bdj.4814073.

[42] B. Holme, L.H. Hove, A.B. Tveit, Using white light interferometry to measure etching of dental enamel, Measurement. 38 (2005) 137-147. doi:10.1016/j.measurement.2005.04.003. 
[43] F. Pascaretti-Grizon, G. Mabilleau, M.F. Basle, D. Chappard, Measurement by vertical scanning profilometry of resorption volume and lacunae depth caused by osteoclasts on dentine slices, J. Microsc. 241 (2011) 147-152. doi:10.1111/j.1365-2818.2010.03410.x.

[44] F. Pascaretti-Grizon, G. Mabilleau, D. Chappard, Abrasion of 6 dentifrices measured by vertical scanning interference microscopy, J. Appl. Oral Sci. Rev. FOB. 21 (2013) 475481. doi:10.1590/1679-775720130204.

[45] A.-M. Paepegaey, M.L. Barker, D.W. Bartlett, M. Mistry, N.X. West, N. Hellin, L.J. Brown, P.G. Bellamy, Measuring enamel erosion: a comparative study of contact profilometry, non-contact profilometry and confocal laser scanning microscopy, Dent. Mater. Off. Publ. Acad. Dent. Mater. 29 (2013) 1265-1272. doi:10.1016/j.dental.2013.09.015.

[46] I. Elsayad, A. Sakr, Y. Badr, Combining casein phosphopeptide-amorphous calcium phosphate with fluoride: synergistic remineralization potential of artificially demineralized enamel or not?, J. Biomed. Opt. 14 (2009) 44039. doi:10.1117/1.3210780.

[47] G.J. Huang, B. Roloff-Chiang, B.E. Mills, S. Shalchi, C. Spiekerman, A.M. Korpak, J.L. Starrett, G.M. Greenlee, R.J. Drangsholt, J.C. Matunas, Effectiveness of MI Paste Plus and PreviDent fluoride varnish for treatment of white spot lesions: A randomized controlled trial, Am. J. Orthod. Dentofacial Orthop. 143 (2013) 31-41. doi:10.1016/j.ajodo.2012.09.007.

[48] Balan A., Andrian S., Savin C., Sandu A.V., Petcu A., Stoleriu S., Comparative study regarding the effect of remineralizing products on primary teeth dissolution induced by acidic drinks., Rev. Chim. 66 (2015) 562-564.

[49] O.A. Adebayo, M.F. Burrow, M.J. Tyas, An SEM evaluation of conditioned and bonded enamel following carbamide peroxide bleaching and casein phosphopeptide-amorphous calcium phosphate (CPP-ACP) treatment, J. Dent. 37 (2009) 297-306. doi:10.1016/j.jdent.2008.12.005.

[50] C. Poggio, M. Lombardini, P. Vigorelli, M. Ceci, Analysis of dentin/enamel remineralization by a CPP-ACP paste: AFM and SEM study, Scanning. 35 (2013) 366-374. doi:10.1002/sca.21077.

[51] P.H. De Campos, M.E. Sanabe, J.A. Rodrigues, D.A. Duarte, M.T.B.R. Santos, R.O. Guaré, cristiane Duque, A. Lussi, M.B. Diniz, Different bacterial models for in vitro induction of non-cavitated enamel caries-like lesions: Microhardness and polarized light miscroscopy analyses: BACTERIAL MODEL for CARIES LESIONS INDUCTION, Microsc. Res. Tech. 78 (2015) 444-451. doi:10.1002/jemt.22493.

[52] W.T.A. AWARE, T.P.S. DOCUMENTATION, Implants for surgery-In vitro evaluation for apatite-forming ability of implant materials, (2007).

[53] J.-Y. Gal, Y. Fovet, M. Adib-Yadzi, About a synthetic saliva for in vitro studies, Talanta. 53 (2001) 1103-1115. doi:10.1016/S0039-9140(00)00618-4.

[54] A. Kumar, J. Reed, G. Sant, Vertical Scanning Interferometry: A New Method to Measure the Dissolution Dynamics of Cementitious Minerals, J. Am. Ceram. Soc. 96 (2013) 27662778. doi:10.1111/jace.12482.

[55] I. Pignatelli, A. Kumar, K.G. Field, B. Wang, Y. Yu, Y.L. Pape, M. Bauchy, G. Sant, Direct Experimental Evidence for Differing Reactivity Alterations of Minerals following Irradiation: The Case of Calcite and Quartz, Sci. Rep. 6 (2016) 20155. doi:10.1038/srep20155. 
[56] I. Pignatelli, A. Kumar, M. Bauchy, G. Sant, Topological control on silicates' dissolution kinetics, Langmuir 32 (2016) 4434-4439.

[57] S.V. Dorozhkin, Dissolution mechanism of calcium apatites in acids: A review of literature, World J. Methodol. 2 (2012) 1-17. doi:10.5662/wjm.v2.i1.1.

[58] J.D.B. Featherstone, A. Lussi, Understanding the chemistry of dental erosion, Monogr. Oral Sci. 20 (2006) 66-76. doi:10.1159/000093351.

[59] R.P. Shellis, M.E. Barbour, S.B. Jones, M. Addy, Effects of $\mathrm{pH}$ and acid concentration on erosive dissolution of enamel, dentine, and compressed hydroxyapatite, Eur. J. Oral Sci. 118 (2010) 475-482. doi:10.1111/j.1600-0722.2010.00763.x.

[60] M. Finke, K.D. Jandt, D.M. Parker, The Early Stages of Native Enamel Dissolution Studied with Atomic Force Microscopy, J. Colloid Interface Sci. 232 (2000) 156-164. doi:10.1006/jcis.2000.7200.

[61] J.D.B. Featherstone, C.P. Shields, B. Khademazad, M.D. Oldershaw, Acid Reactivity of Carbonated Apatites with Strontium and Fluoride Substitutions, J. Dent. Res. 62 (1983) 1049-1053. doi:10.1177/00220345830620100801.

[62] M.J. Larsen, A. Richards, Fluoride is unable to reduce dental erosion from soft drinks, Caries Res. 36 (2002) 75-80. doi:57595.

[63] A. Vieira, D.H.J. Jager, J.L. Ruben, M.C.D.N.J.M. Huysmans, Inhibition of erosive wear by fluoride varnish, Caries Res. 41 (2007) 61-67. doi:10.1159/000096107.

[64] G.F. Ferrazzano, M. Coda, T. Cantile, G. Sangianantoni, A. Ingenito, SEM investigation on casein phosphopeptides capability in contrasting cola drinks enamel erosion: an in vitro preliminary study, Eur. J. Paediatr. Dent. Off. J. Eur. Acad. Paediatr. Dent. 13 (2012) 285288.

[65] M.T. Pulido, J.S. Wefel, M.M. Hernandez, G.E. Denehy, S. Guzman-Armstrong, J.M. Chalmers, F. Qian, The inhibitory effect of MI paste, fluoride and a combination of both on the progression of artificial caries-like lesions in enamel, Oper. Dent. 33 (2008) 550-555. doi:10.2341/07-136.

[66] A. Al-Mulla, L. Karlsson, S. Kharsa, H. Kjellberg, D. Birkhed, Combination of highfluoride toothpaste and no post-brushing water rinsing on enamel demineralization using an in-situ caries model with orthodontic bands, Acta Odontol. Scand. 68 (2010) 323-328. doi:10.3109/00016357.2010.512863.

[67] A. Nordström, D. Birkhed, Preventive effect of high-fluoride dentifrice $(5,000 \mathrm{ppm})$ in caries-active adolescents: a 2-year clinical trial, Caries Res. 44 (2010) 323-331. doi:10.1159/000317490.

[68] J.M. ten Cate, M.J. Buijs, C.C. Miller, R. a. M. Exterkate, Elevated fluoride products enhance remineralization of advanced enamel lesions, J. Dent. Res. 87 (2008) 943-947.

[69] N.R. Mohammed, R.J.M. Lynch, P. Anderson, Effects of fluoride concentration on enamel demineralization kinetics in vitro, J. Dent. 42 (2014) 613-618. doi:10.1016/j.jdent.2013.12.005.

[70] Y. Iijima, F. Cai, P. Shen, G. Walker, C. Reynolds, E.C. Reynolds, Acid resistance of enamel subsurface lesions remineralized by a sugar-free chewing gum containing casein phosphopeptide-amorphous calcium phosphate, Caries Res. 38 (2004) 551-556. doi:10.1159/000080585.

[71] R.K. Rose, Effects of an anticariogenic casein phosphopeptide on calcium diffusion in streptococcal model dental plaques, Arch. Oral Biol. 45 (2000) 569-575. 
[72] R.K. Rose, Binding characteristics of Streptococcus mutans for calcium and casein phosphopeptide, Caries Res. 34 (2000) 427-431. doi:16618.

[73] K.H. Lee, H.I. Kim, K.H. Kim, Y.H. Kwon, Mineral loss from bovine enamel by a $30 \%$ hydrogen peroxide solution, J. Oral Rehabil. 33 (2006) 229-233. doi:10.1111/j.13652842.2004.01360.x.

[74] C. Poggio, M. Lombardini, A. Dagna, M. Chiesa, S. Bianchi, Protective effect on enamel demineralization of a CPP-ACP paste: an AFM in vitro study, J. Dent. 37 (2009) 949-954. doi:10.1016/j.jdent.2009.07.011.

[75] Q. Zhang, J. Zou, R. Yang, X. Zhou, Remineralization effects of casein phosphopeptideamorphous calcium phosphate crème on artificial early enamel lesions of primary teeth, Int. J. Paediatr. Dent. Br. Paedodontic Soc. Int. Assoc. Dent. Child. 21 (2011) 374-381. doi:10.1111/j.1365-263X.2011.01135.x. 